\title{
A HYBRID APPROACH TO WORKFLOW MODELLING
}

\author{
Richard Cull, CMA \\ Tillal Eldabi, $\mathrm{PhD}$
}

\author{
Brunel Business School, Brunel University, London, UK \\ \{richard.cull; tillal.eldabi\}@brunel.ac.uk
}

\begin{abstract}
The increase in Business Process Management projects in the past decade has seen an increase in demand for business process modelling techniques. A rapidly growing aspect of BPM is the use of workflow management systems to automate routine and sequential processes. Workflows tend to move away from traditional definitions of business processes can often be forced to fit a model which does not suit its nature. Existing process modelling tools tend to be biased to either the informational, behavioural or object oriented aspect of the workflow. Because of this, models can often miss important aspects of a workflow. As well as managing the relationship between the types of model it is important to consider who will be using it as process models are useful in various ways. This paper reports on a case study in a manufacturing company where users were surveyed to see which are the notation that are most common in modelling based on two main categories (behavioural and informational). Research outcomes showed that there is no prevailing set of standards used for either of these categories, whilst most user feel the need to use more than one approach to model their system at any given time.
\end{abstract}

Keywords: Business Process Management, Process Modelling, BPMN, Workflow

\section{INTRODUCTION}

\subsection{Research Problem}

Business Process Management (BPM) is a concept which has been growing rapidly since the late 1990s (Lu and Sadiq 2007; Melao and Pidd 2000). Generally, the BPM lifecycle as described by zur Muehlen et al (2006) consists of process discovery, specification, implementation, execution, monitoring and controlling and if used correctly can improve the way a company conducts its business in the long and the short term (Sinur 2003, Rausch 2006). BPM is often seen as the follow up to Business Process Re-engineering (Hammer 1990, Davenport 1993 and Hammer and Champy 1993) and before that Total Quality Management philosophy. BPR is a radical, revolutionary approach to business process improvement whilst TQM is incremental, evolutionary and continuous in nature. It can be argued BPM integrates TQM and BPR by using a re-engineering approach to improve business quality (Hung 2006). The increase in its use has lead to a significant rise in the amount of BMP Software (BPMS) systems that are commercially available (Lu and Sadiq 2007; Melao and Pidd 2000).

The research in this paper will focus on only one kind of BPMS package, Workflow Management Systems (WfMS). The increasing interest in BPM and Workflow packages has raised a need to standardise concepts and practices (Mohan 1997). For example, the Workflow Management Coalition (WfMC) was founded in order to achieve this objective and has made an effort to oversee this standardisation process and has already compiled a set of standards for WfMSs. However it has been noted that although WfMC has been successful to a certain extent, however, they have struggled to implement standard business process modelling concepts (van der Aalst 
2003, van der Aalst et al 2005). The two main obstacles to that are the amount of specifications that are being proposed and that too many standards are being proposed with a commercial interest, meaning they are geared towards producing benefits to a new product.

Process modelling is becoming increasingly important as the focus shifts to BPM and there are so many available techniques and standards that it is difficult for businesses to determine which notation suits their needs. One influential development in the field of workflow was the identification of the "workflow patterns" proposed by van der Aalst et al (2003). These patterns focus on both imperative and optional routing constructs which can govern the path a workflow may take. They range from basic control patterns such as sequence flow and exclusive choice gateways to the more complex structural patterns such as arbitrary cycles. These patterns have become the main foundation for the development and evaluation of many new process modelling notations including, but not limited to, Business Process Management Notation and UML Activity Diagrams, two of the most popular modelling notations (White 2005, van der Aalst 2003). The WfMC and leading authors in the field of workflow management (most notably Wil van der Aalst who has contributed much research to the subject) often propose Petri Nets (Petri 1962) as the most suitable tool for modelling workflows. However, Petri Nets - although have been subject to much research over the years - are still being used very little in practice (Bray 2002). A major factor for this could be the fact they are mathematically complex and although they are graphical, are not always easy to read (Desel 2001). However in addition to this problem, many of the workflow patterns (van der Aalst et al 2003), cannot be mapped onto Petri nets easily which emphasises the importance of these patterns.

The lack of a standard approach to workflow modelling can lead to confusion and not having a good process model can severely damage the success of a workflow project. Van der Aalst (2005) stated that this lack of standardisation had created a "horses for courses" approach and indicated that a solution should use "well-established process modelling techniques". Of these "wellestablished" graphical techniques there are still no standard approaches. The most prominent modelling approaches are Petri Nets, Business Process Management Notation, (BPMN), UML Activity Diagrams, Role Activity Diagrams (RAD), Data Flow Diagrams (DFDs) and StateTransition Diagrams (STDs) however each notation does not map a process sufficiently on its own. Notations like BPMN, UML and DFDs focus on the informational perspective of a process (e.g. the tasks and information flow involved in a process). While notations such as RADs and STDs focus on the behavioural aspect of a process (e.g. concerned with how the user does something and how an action changes the state of the users and the system). Neither of these is more important than the others which means that using a model from one perspective will have an opportunity cost by nor using the others. For example, using an informational model means we lose some behavioural perspective and vice-versa. Although we have several informational models and behavioural models to choose from we do not have the option of modelling the entire process using just one notation. Without identifying both aspects of the process we cannot hope to get the full picture.

\subsection{Research aims and objectives}

The aim of this paper is to investigate and assess the feasibility and potential benefits of using hybrid modelling approaches to represent workflow processes and aid BPM projects and use the results as the specification for a hybrid modelling notation.

In order to achieve this aim it would be necessary to assess the various prevalent modelling methodologies that are already available and determine their strengths and weaknesses. It is 
hoped that outcomes of this research should act as both justification and guidance into what the hybrid model notation should accomplish. In general, it should have the following attributes:

- Able to model the information flow and routing constructs and to handle external events and human actors i.e. model the workflow's informational and behavioural aspects.

- The notation should be potentially scalable so that it can be used for different purposes (e.g. simulation, execution, user training etc.)

- Use a notation that is recognisable from other popular methodologies so that it is easy to use for all type of user.

\section{A BRIEF OVERVIEW OF WORKFLOW}

WfMSs are designed to automate routine, sequential business processes. Usually they involve the movement of paper documents throughout an office such as a company's purchase invoice procedure or their holiday card process. They move away from the traditional "input/output" definition provided by Hammer and Champy (1993). In most cases workflows are fairly rigid and predictable, the introduction of sterner government legislation such as the Sarbanes Oxley Act of 2002 have made this more so. WfMSs will normally be based upon the same principles as shown in the Workflow Reference Model (Figure 1), and detailed below that.

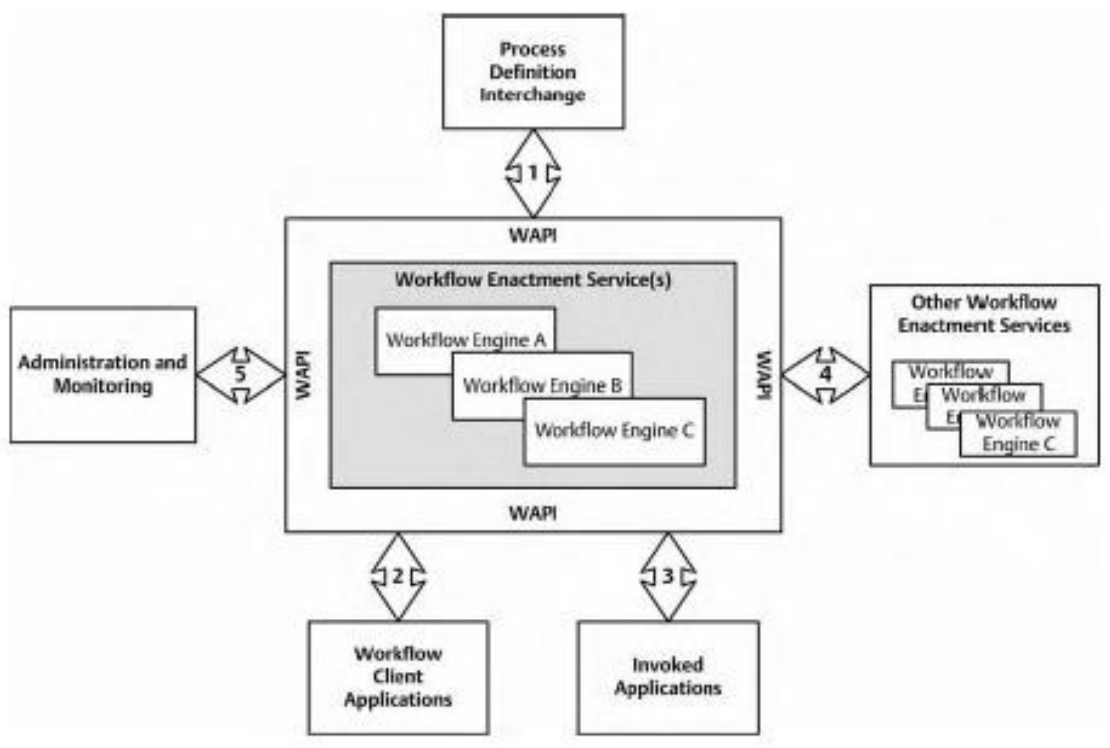

Figure 1. WfMC Workflow Reference Model, The Workflow Reference Model, Workflow Management Coalition (1995)

- Workflow Definition Tools - These tools provide the process modeller and developer with all the necessary means to build a workflow. This includes building the process model, the tasks and activities involved as well as any user roles in the process. The workflow definition tools are usually graphic based and allow the developer to build the workflow as he creates the process model.

- Workflow Client Application - The workflow client is the end of the system which is presented to the user. From here they can perform the tasks which are assigned to them as defined by the developer in the process definition tools. Each workflow instance is presented to the user in the form of a "worklist" or "to-do list" which displays each instance of a workflow as a separate item of work. 
- Workflow Engine - The workflow engine is responsible for interpreting the workflow definition as specified by the developer and manages the execution of individual workflow instances and the building of user's worklists.

- Often the WfMS will run with a database back-end where all of the data relating to each workflow and each of its instances is stored.

WfMS engines will be able to handle various routing constructs, ranging from simple sequence flow to complex conditional splits. The work of Wil van der Aalst (2003) has identified 43 of these "workflow patterns". These are often used to measure the validity of WfMSs and workflow modelling notations, for example Stephen White's paper on BPMN which compared its representations of the workflow patterns against that of UML activity diagrams (White 2005). By constructing the model based on these patterns we can build the process using the definition tools and allow the engine to interpret and manage the workflow rules. However, although WfMSs allow us to automate a business process they cannot handle certain external actions undertaken by staff and the process model should always take into account human actors, a factor which is often neglected among workflow research.

The workflow definition tool will allow the developer to build the workflow application often using some form of graphical modelling notation, often one of the commercial and product specific "horses-for-courses" notations van der Aalst described. The developer will then build logic into the graphical model to be interpreted by the engine. As well as graphical models, an emerging approach is to use executable models. These models are usually written in some form of pseudo-code and are created with the aim of completing simulation runs of the process. Probably the most prominent executable modelling specification in the research is Business Process Execution Language (BPEL). BPEL is the executable extension to BPMN and the two can be converted into the other. This approach gives us the option of both graphical and executable modelling. Complex, executable models are valuable to process design and analysis but modelling approaches should be designed at covering the entire process and all business users. BPEL is unlikely to be useful to anyone other than the analysts and developers while BPMN was designed in such a manner that analysts, developers and also business users should be able to comprehend the model (White 2004). Because of this BPMN has been successfully implemented in BPM projects and has seen much research (Owen et al 2003; van der Aalst 2006 and 2003). The scalability of the model, with regards to the amount of people from different backgrounds that can find the model useful and the other modelling languages it can interact with, is equally as important as its perspective.

\section{RESEARCH METHODOLOGY}

\subsection{Research Design}

To achieve the objectives of the research it was important to first identify popular modelling notations from both the informational and behavioural perspective. By reviewing popular modelling techniques we are ensuring that the specification which arises from the research is both recognisable to end users and easy to use. In order to identify which techniques should be included it will be necessary to try to get an idea as to which notations people are familiar with. This was done by using observational research to gather the opinions of people within a business with varying levels of skills, competencies and experiences. This involved setting an example assignment to the group and discussing the results with each individual. Using this technique it was possible to identify common characteristics that belonged to certain informational and behavioural notations. The second part of the research was to evaluate these techniques and the advantages and disadvantages of using them. The final step was to collate the results and use the 
findings to build the foundations of the specification. Again following the principles of action research the group of users will be given process models generated using the four techniques from the first study and asked to review their effectiveness. This will again be conducted among various skill and competence levels

\subsection{Participants}

For the first phase of the research it was important to select a group of people with varying skill levels. A small case study was conducted within a manufacturing company with users from various backgrounds. The company is one of the largest manufacturer of excavators and building equipment in the world and has recently started a BPM project using the Workflow platform Metastorm $^{\mathrm{TM}}$ BPM. Eight members of the project team are asked to participate in the research. The decision on who formed the team was based upon their role and their experience. It was important to identify a set of users with a varied set of responsibilities and experience in order to assess the results fairly. This sample is complimented by interviews with the vendor of their WfMS, Metastorm. One of the packages in their BPM suite is a tool called Metastorm ProVision ${ }^{\mathrm{TM}}$. This tool allows the user to model the process using a wide variety of notations. Metastorm are an ideal choice of subject because they are a market leader with over 10 years of experience in BPM systems. Their wide range of clients, in terms of both market and size, means that information fed back into the research is not only from a large sample, but also from a diverse one. By conducting research into how this tool is used with Metastorm we can ascertain which notations appear to be more popular.

The second phase of the research we will apply four popular techniques to a pilot process in a real project within the company (Action Research) to see how notations will be perceived. From this we can identify the problems which some of the popular techniques contain. In order to avoid bias within the results all participants were tested individually without being able to consult the other subjects on what they should say. All names of researchers, participants and modelling techniques were removed during the tests until after the results had been collated.

\subsection{Evaluating the Results}

The first part of the research was to ask eight members of the company's IT department to build a process model for a fictional "New Hire" process. This was a fairly small workflow but the process was specified in such a manner that it included some mildly complicated routing constructs. The basis of the process design is that it would involve both discriminatory (the flow will go one route out of a number of potential options depending on a condition) and indiscriminate (the flow will split into a number of routes in parallel) routing constructs. The purpose of this task is to assess the notation that people expect to see used for certain components of the business process. The results of this will be useful to define common features that the hybrid specification should include.

The second part of this step was to work together with Metastorm ProVision and assess both the company's and their customer's expectations from workflow modelling. Metastorm ProVision ${ }^{\mathrm{TM}}$ is a tool which is provided to customers of Metastorm ${ }^{\mathrm{TM}}$ BPM to design their business processes before they are built using their WfMS. ProVision not only allows the user to design workflows using a number of business process modelling techniques including BPMN, IDEF, UML and Six Sigma but also allows them to create their own notations from a combination of existing tools. Together with Metastorm a survey is conducted into which modelling notations are most popular, which techniques users would like to see implemented in future and how many create their own notation. The results will indicate which of the available notations are most successful in the eyes 
of end users as well as highlighting the popular notations which are not available in ProVision and also re-emphasising the need to combine these techniques to solve the informational/behavioural divide. The result of these two investigations allows us to identify two techniques from each perspective to apply to the next stage which is evaluating their usefulness.

In order to assess the usefulness of the four selected notations we will again as the company to apply each of these techniques to the "New Hire" process. Each business analyst will be asked to model the new hire process using each model. Each of these four models will be passed to the three developers who will develop the process based on each model. Each workflow will then be released to an end user who will be asked to review each of the workflows using the process models as a help guide. Feedback on the advantages and disadvantages of each model will be taken at each stage. The analyst will be asked to describe how easy the modelling notation is to use and to apply to a workflow process. The developer will be asked how easy the model is to understand and how easy it was to apply to a WfMS. The end user will be asked how easy the model was to read and how well it represented the final workflow. Finally once the results have been assessed a specification for the hybrid notation can be proposed.

\section{RESEARCH FINDINGS}

\subsection{Phase 1}

In the first phase of the research the project team at the company were asked to create a model for their pilot process using whatever notation they were used to. The pilot process chosen was the company's procedure for hiring new staff. The results of the investigation were remarkably similar. Of the eight participants in the research, three were business analysts, three were developers and two were managers or seniors. The first two components which were checked were tasks and actions. These components can describe the full process at a high level. The results showed that every subject agreed that the expected notation for a task was a rectangle containing a description of the task and that an action is expected to be a line with an arrowhead. This is usually the case with informational models, with some minor differentiations.

The next four components had more complicated routing constructs. The first was a discriminatory split or a decision point. In this case the workflow can only take one of several conditional directions. In such case the decision is whether or not the new member of staff is a member of production or the office. If he/she is an office worker, notification should be sent to IT to create a new account. If he/she is production he/she does not have access to a computer terminal and an IT is not required. Each subject modelled the decision point using a diamond which is the most common method of modelling a decision (referred to as an OR-SPLIT). In addition to an OR-SPLIT the subjects had to model a parallel split (AND-SPLIT). An ANDSPLIT differs from the OR-SPLIT in that the workflow will go down both paths. In the example of the new hire process, if a new member of staff is an office worker, notification will be sent to payroll, to allocate an employee number, and to IT, to create their new account. Some of the subjects did not discriminate between an AND-SPLIT and the OR-SPLIT and used the diamond notation. Others just showed two actions leaving the task and going in two directions.

The final two components which the subjects were asked to model were almost always ignored. These were behavioural aspects of the workflow. Every subject ignored the behavioural aspects of the process apart from one. First the subtasks within the new hire process were represented by the modeller with the same notation as a regular task but with a small "plus" symbol at the bottom. This notation is familiar to BPMN. A subtask in this instance is a task or process which is triggered by the main process but does not have any direct impact on it. In this instance the 
subtasks were the process of creating a new IT account and the process of creating a new payroll account. These two processes are separate to the new hire process and so should be modelled differently.

The next behavioural aspect was the concept of user states. When the new member of staff's manager has raised a new hire form he/she will then become inactive in the process. However he is unlikely to be completely inactive in the business. After he raises the form the manager might have other tasks to do related to but not integral to the process (i.e. allocate a desk, brief the rest of the department on the new starter etc.) One subject did in fact model this change in state. The notation used was a circle containing a description of the state changed. Table 1 shows all of the results of the action research project - phase 1 .

Table 1: Results of Action Research project - phase 1

\begin{tabular}{|c|c|c|c|c|c|c|}
\hline Subject & Task & Action & OR-SPLIT & $\begin{array}{l}\text { AND- } \\
\text { SPLIT }\end{array}$ & Subtask & User State \\
\hline 1 & Rectangle & $\begin{array}{c}\text { Straight } \\
\text { Line with } \\
\text { arrowhead }\end{array}$ & Diamond & Diamond & None & None \\
\hline 2 & Rectangle & $\begin{array}{c}\text { Straight } \\
\text { Line with } \\
\text { arrowhead }\end{array}$ & Diamond & Diamond & None & None \\
\hline 3 & Rectangle & $\begin{array}{l}\text { Straight } \\
\text { Line with } \\
\text { arrowhead }\end{array}$ & Diamond & $\begin{array}{c}\text { Two } \\
\text { separate } \\
\text { lines with } \\
\text { arrowheads }\end{array}$ & None & None \\
\hline 4 & Rectangle & $\begin{array}{l}\text { Straight } \\
\text { Line with } \\
\text { arrowhead }\end{array}$ & Diamond & $\begin{array}{c}\text { Two } \\
\text { separate } \\
\text { lines with } \\
\text { arrowheads }\end{array}$ & None & None \\
\hline 5 & Rectangle & $\begin{array}{l}\text { Straight } \\
\text { Line with } \\
\text { arrowhead }\end{array}$ & Diamond & $\begin{array}{c}\text { Diamond } \\
\text { containing } \\
\text { plus } \\
\text { symbol }\end{array}$ & $\begin{array}{c}\text { Rectangle } \\
\text { containing } \\
\text { plus } \\
\text { symbol }\end{array}$ & Circle \\
\hline 6 & Rectangle & $\begin{array}{l}\text { Straight } \\
\text { Line with } \\
\text { arrowhead }\end{array}$ & Diamond & $\begin{array}{l}\text { Diamond } \\
\text { containing } \\
\text { plus } \\
\text { symbol }\end{array}$ & $\begin{array}{c}\text { Rectangle } \\
\text { containing } \\
\text { plus } \\
\text { symbol }\end{array}$ & None \\
\hline 7 & Rectangle & $\begin{array}{l}\text { Straight } \\
\text { Line with } \\
\text { arrowhead }\end{array}$ & Diamond & $\begin{array}{c}\text { Two } \\
\text { separate } \\
\text { lines with } \\
\text { arrowheads }\end{array}$ & None & None \\
\hline 8 & Rectangle & $\begin{array}{l}\text { Straight } \\
\text { Line with } \\
\text { arrowhead }\end{array}$ & Diamond & $\begin{array}{c}\text { Two } \\
\text { separate } \\
\text { lines with } \\
\text { arrowheads }\end{array}$ & None & None \\
\hline
\end{tabular}

The informational notations arose from the research are very similar to those of BPMN and UML activity diagrams. The behavioural notations were often missed so it is difficult to ascertain which notations are most recognisable. The notation used for the state change however is similar to that used in state transition diagrams and activity cycle diagrams. 
Looking at Metastorm ProVision we found that process modelling notations that were most frequently used are BPMN; this was followed by UML Activity Diagrams, Six Sigma and IDEF. The research from the package also indicated that many customers preferred to use the tool to create their own notations. This highlights the fact that using just one of these techniques is not solely sufficient for many BPM projects. However by leaving companies to build their own modelling standards and notations, we will negate the original points of having a standard notation system.

\subsection{Phase 2}

The results of the first two tests meant it seemed sensible to use BPMN and UML Activity Diagrams for the final tests. Their notation is familiar to all and they are probably market leaders in informational modelling at the moment. In addition to this there is already a vast amount of literature supporting and comparing these two techniques.

The lack of behavioural models being used points to the fact that there are no such popular notations which allow us to model this perspective whilst also retaining an adequate representation of the informational side. Because most behavioural models give a limited informational perspective they cannot accurately describe a workflow. This again re-emphasised the need for a hybrid methodology. The models which were chosen for the experiment were Role Activity Diagrams and Activity Cycle Diagrams. RADs were chosen because they are a growing methodology among the research community which are aimed at solving a similar problem to that which is presented in this paper and it could be that RADs could be modified slightly to meet the aim and objectives in Section 1.2 Activity Cycles were chosen mainly because their notation for changing states and new tasks is the same as that used by subject 5 in the initial test but also because of their usefulness in simulation. One of our objectives in Section 1.2 was to make a specification that has the potential to be used in various ways such as simulation and using activity cycle diagrams could help us achieve this.

Four process models were created and passed to the project team at the company. The aim of this phase was to create a workflow application using each of the four models and review the results of each. This would help us identify the strengths and weaknesses of each technique. Again people from different backgrounds were involved in the research and so had different requirements for the model. The results would be influenced by the motives of each business user.

The workflow was created by the project team using each model and the accuracy at each step was measured. Each member was asked to give their comments on the notation and rank each notation giving the rank one to the notation they found most useful and four to the notation they found least useful. The highest ranked notation was awarded three points, second highest was awarded two points and so on.

The project team was split into four functional groups; managers, analysts, developers and endusers. Managers appeared to prefer the informational models as BPMN scored highest followed by Activity Diagrams. These models present the workflow structure at a high level and are easy for someone who has limited experience or involvement with the process to get an understanding of the functional bands and information flow. For this purpose, informational models are suitable on their own however the business analysts on the team commented that using a combination of both BPMN and RADs allowed them to model the process in its entirety and identify parts of the process that BPMN had not. These included the work partitioning of tasks and subtasks and 
passive user states. Developers' preferences were the same as those of the managers. They commented that they only needed to understand the process at a high level and that as they had the informational level workflow they could build a working version although they did use the information in the activity cycle diagram as well. End users showed a preference for the informational/behavioural mix as the behavioural models show better how they should be working rather than what they should be doing. The behavioural aspects of the flow better describe how the process works rather than stating what it should do. The results of the action research project - phase 2 can be found in Table 2.

Table 2: Results of Action Research project - phase 2

\begin{tabular}{|c|c|c|c|c|}
\hline Subject & BPMN & $\begin{array}{c}\text { UML Activity } \\
\text { Diagram }\end{array}$ & $\begin{array}{c}\text { Role Activity } \\
\text { Diagram }\end{array}$ & $\begin{array}{c}\text { Activity Cycle } \\
\text { Diagram }\end{array}$ \\
\hline 1 & 1 & 2 & 4 & 3 \\
\hline 2 & 1 & 2 & 3 & 4 \\
\hline 3 & 2 & 4 & 1 & 3 \\
\hline 4 & 1 & 4 & 3 & 3 \\
\hline 5 & 1 & 2 & 4 & 3 \\
\hline 6 & 1 & 2 & 4 & 2 \\
\hline 7 & 1 & 3 & 4 & 1 \\
\hline 8 & 2 & 3 & 4 & 12 \\
\hline Score & 22 & 10 & 5 & 3 \\
\hline
\end{tabular}

The results indicated that BPMN was found preferable for the informational notations and activity cycle diagrams were found preferable for the behavioural notations. The comments made during the research indicated that this was mostly down to the usability and ease of use of the notation. People were often confused by the Role Activity Diagrams and their emerging notation, preferring instead the simplistic notation of the Activity Cycle Diagrams. Likewise with the informational models, the BPMN notation was often deemed easier to use than that of Activity Diagrams.

\section{DISCUSSION}

The results of the research indicate that many companies choose to combine one or more popular modelling techniques. This was indicated by the results of the investigation into how customers of Metastorm BPM use the modelling package Metastorm ProVision and by the results of the action research case study at the company. This suggests that a standard for hybrid modelling could be useful for workflow projects as it removes the need to duplicate effort put into building two models. Action research at the company confirmed that users in different business functions will have different requirements for a business process model and may prefer different models to the choice of other functional bands.

These points indicate that the potential benefits of having a more structured hybrid modelling approach are well placed. The research suggests that BPMN is a suitable modelling tool for depicting the informational side of the process. Its features were recognisable to most of the participants of the observational case study and proved to be the most popular technique among Metastorm customers. Hence, it would be wise to consider using features of BPMN when creating the specification for the hybrid model notation. UML activity diagrams also scored reasonably well but did not seem to be as popular as BPMN. However that is not to say that no features from this approach will be considered in the specification. Users actually preferred the 
"fork and join" nodes used in UML Activity Diagrams to the full BPMN notation for a parallel split. Using BPMN notation also means the hybrid specification has the potential to integrate with BPEL which helps us to achieve one of the objectives of this paper which was that the notation considered should be scalable.

The results of the research for behavioural models suggested that users were comfortable with the notation for activity cycle diagrams. This technique is mostly used for simulation which means that if parts of this notation are included in the hybrid specification it could eventually be extended to simulation modelling. This falls in line with the objective of identifying a scalable notation. Role Activity Diagrams were not well received during the research. Despite the fact that they do achieve modelling both informational and behavioural levels of the process well, their notation was considered to confusing by subjects of the action research group. This was quite a surprising outcome as RADs were expected to receive the most positive feedback because of their innovative notation, however this was deemed as a disadvantage by the research subjects.

These results allow us to move on to the next phase which is to formally build the framework for the hybrid modelling notation based on the more popular techniques discussed in this paper. The notations used within the hybrid specification will not only allow us to model a process at both the informational and behavioural level but also create the potential to extend the model to simulation and executable modelling.

\section{CONCLUSIONS}

This paper has discussed the fact that existing techniques do not allow users to model both the informational and behavioural perspectives of a process. In order to identify potential notations for the hybrid model we asked business users from various levels of skill and competency to model a fictional process based on their experiences and expectations. The results of this showed us the different graphical symbols that business users are used to seeing in process diagrams. We also liaised with a leading WfMS distributor, Metastorm ${ }^{\mathrm{TM}}$, to identify those techniques which are popular with customers of their BPM suite. After reviewing the results of this research it was indicated that current trends lean towards the informational perspective of process and that behavioural aspects of time, state changes and business environment were often being neglected.

Using the results of the research process models were generated for a fictional process using four different notations which fit user expectations. These models were passed between users in varying job roles and skill levels to build a sample workflow. The accuracy of each model was compromised because it was based on informational or behavioural theories only.

The results of the research gave an indication of the perceptions and expectations that business users have with regards to business process modelling. The findings will help to bridge the gap between the theory behind the model notations and making them suitable for business users. From this small study it seems that business users have a preference for the informational style process models so the challenge is to portray behavioural detail in such a manner that the look and feel of the model is not compromised. A potential investigation into extensions to the BPMN language is suggested by its high score in the results of the survey. The sample included in this study is not large enough - albeit informative - to draw any concrete conclusions and so the next step is to refine the notation based on this specification. Further work will include developing the modelling notation from the results of this paper into a full process modelling specification. Future revisions of the notation should be applied and tested against several BPM case studies to measure its accuracy. Future projects could also include mapping the notation to an executable modelling language such as BPEL and extending the notation for use in simulation. 


\section{REFRENCES}

1. Lu, R., Sadiq, R.W. (2007). A Survey of Comparative Business Process Modelling Approaches. BIS 2007: 82-94

2. Melao, N., Pidd, M. (2000). A Conceptual Framework for Understanding Business Processes and Business Process Modelling. Information Systems Journal, Vol.10, No. 2.

3. zur Muehlen, M., Ho, D.T. (2006). Risk Management in the BPM Lifecycle. In Proc. Business Process Management Workshops (BPM2005), LCNS, Vol. 3812 (2006) 454-466

4. Sinur, J., Thompson, J. (2003). The business process management scenario. Gartner Article Top View $A V-20-0932$,

5. Rausch, T. (2006). Holistic Business Process and Compliance Management. Conference for Systems Integration 2006

6. Hammer, M. (1990), "Re-engineering work: Don't automate, obliterate", Harvard Business Review, Vol. 68 No.4, pp.104-12.

7. Davenport, T. (1993). Process Innovation: Reengineering work through information technology, Harvard Business School Press, Boston

8. Hammer M, Champy J. (1993) Reengineering the corporation: a manifesto for business revolution. New York: Harper Business Editions

9. Hung, R. Y. -Y. (2006). Business Process Management as Competitive Advantage: a review and empirical study. Total Quality Management, 17(1), 2-40.

10. Mohan, C. (1997), Recent Trends in Workflow Management Products, Standards and Research. IBM Almaden Research Center

11. van der Aalst, W.M.P., ter Hofstede, A.H.M., and Weske M. (2003). Business Process Management: A Survey. In: Proceedings of BPM'2003. pp 1-12. Springer LNCS 2678. Heidelberg: 2003

12. van der Aalst, W.M.P., ter Hofstede, A.H.M. (2005). YAWL: Yet Another Workflow Language. Information Systems 30 (2005) 245-275

13. Petri, C.A. (1962) Kommunikation mit Automaten, Ph.D. Thesis, Fakultat fur Mathematik und Physik, Technische Hochschule Darmstadt, Darmstadt, Germany.

14. Ian K Bray, (2002), An Introduction to Requirements Engineering, Addison Wesley

15. Russell N, ter Hofstede A, van der Aalst W, Mulyar N. (2006). Workflow Control Flow Patterns: A Revised View. Tech. Rep. BPM Centre Report BPM-06-22, BPMcenter.org

16. Stephen A. White, (2004), Introduction to BPMN, IBM Corporation Available at: http://www.bpmn.org/Documents/Introduction\%20to\%20BPMN.pdf

17. Owen M., Raj J., (2003), BPMN and Business Process Management, Popkin Software Available at: http://www.bpmn.org/Documents/6AD5D16960.BPMN_and_BPM.pdf

18. S. White, (2003). Using BPMN to model a BPEL process, BPTrends 3 (3) (2005), pp. 1- 18.

19. Stephen A. White, (2005), Process Modeling Notations and Workflow Patterns, IBM Corporation Available at: http://www.bpmn.org/Documents/Notations\%20and\%20Workflow\%20Patterns.pdf 